\title{
Topology Control with Power and Rate Adaption in Wireless Mesh Networks with QoS Constraint
}

\author{
Kunxiao Zhou, Hui Zhao, and Xing Tang
}

\begin{abstract}
This paper discusses topology control jointing power control problem in wireless mesh networks with QoS guarantee. We are given a set of mesh routers in a plane, some of which are called gateway nodes, as they have wire connection to the Internet. Our task is to construct a routing forest (a set of trees rooted from the gateway nodes), configure the transmission power of each node, such that the constructed network topology can meet the QoS requirement and the system throughput is maximized. We consider rate adaption in this paper. Links can choose an appropriate rate to transmit, according to the channel environments, such as the distance between two end-nodes and the transmission power of the sender. In our proposed method, gateways greedily add routers to its own subtree, in the order of relative distance. A node has a small relative distance to a gateway, if it is closer to this gateway and farther away from all other gateways. In each round, we select the candidate node to be added, and configure transmission power and data transmission rate, such that the QoS requirement is fulfilled and the system total throughput is maximized. Simulation results have shown that our method can significantly improve the network throughput, guaranteeing QoS requirement.
\end{abstract}

Index Terms-Topology control, power control, rate adaptation, QoS, wireless mesh networks.

\section{INTRODUCTION}

Wireless mesh networks (WMNs) [1] attract a lot of research interests in recent years. WMNs have many potential applications, such as last-mile broadband Internet access, neighborhood gaming, and real-time multimedia applications. The throughput is the main concern of WMNs, as all these applications need a high bandwidth. In wireless mesh networks, many applications also require QoS provisions, such as transmission of multimedia data, real-time collaborative work, and interactive distributed applications.

Extensive research has been done on QoS provisions in wireless networks, such as QoS routing or admission control [2]. Most of the existing works deal with resource allocation (e.g., scheduling or buffering) or routing for QoS requests.

Manuscript received July 4, 2016; revised September 19, 2016. This work was supported in part by grants from the Natural Science Foundation of Guangdong Province, China (Grant No. 2014A030310375, 2014A030313632), and the National Natural Science Foundation of China (Grant No. 61402106).

Kunxiao Zhou and Hui Zhao are with the School of Computing, Dongguan University of Technology, Guangdong, China (e-mail: zhoukx1@163.com, jenniferzhao09@gmail.com).

Xing Tang was with School of Computer Science and Technology, Wuhan University of Technology, Hubei, China (e-mail tangxing@whut.edu.cn).
However, the construction of a network topology that can overall meet QoS requirements has not been studied in the literature. In wireless mesh networks, online QoS provisions, such as end-to-end bandwidth and delay, are highly dependent on the network topology. Without a proper configuration of the topology, some nodes in the network could be easily overloaded and it might be impossible to find a QoS route during the operation of the network.

In this paper, we study the problem of joint power and topology control to achieve high throughput for WMNs under QoS constraint. We are given a set of mesh routers, some of which are called gateway nodes, because they have wire connection to the Internet. Supposing each router has a traffic demand from end-uses', our task is to construct a forest (a set of trees rooted from the gateway nodes), assign nodes' transmission power, and route the traffic from mesh nodes to the Internet via the gateway nodes along the forest, such that system total throughput of the network is maximized, satisfying the each node's traffic delivery ratio bound. We consider both uplink traffic and downlink traffic. We assume that the traffic are not splittable, and can only be route to/from Internet through the forest.

We present a joint power and topology control method with rate adaption. In each round, each gateway selects a candidate (node) that has the closest relative distance to this gateway. Each candidate selects a parent node to be connected, and assigns the transmission power for itself and its parent, such that the current system throughput is maximized satisfying QoS bound. We also consider rate adaption. When the transmission power of a link is determined, this link will choose the highest data rate that supports the link connectivity. Among all the candidates selected by gateways in that round, the one with the maximal total throughput will be added to the topology.

The rest of this paper is organized as follows. We discuss the related work in Section II. In Section III, we describe the system model and formulate the problem we concern. The joint power and topology control with QoS guaranteeing method is presented in Section IV. We investigate the performance of our solution through simulations and analyze the results in Section V. Finally, Section VI concludes this paper.

\section{RELATED WORK}

Topology control is widely studied in wireless networks. Its main concern is to construct a topology that fulfill certain requirement, such as low interference, load balancing, or high throughput.

The topology control algorithms that minimize interference 
level can be found in [3], [4]. They expected to minimize the interference level, to improve more spatial reuse, so as to improve the network throughput. Some researches worked on design low node degree topologies to achieve high system throughput. These works may not improve WMN's throughput. Because they prefer to choose small transmission power, and generate networks of large number end-to-end hops. Such a topology is of low throughput, as shown in [5]. In [4], authors proposed an optimization model on the basis of interference and link capacities that found out the aggregate end to end throughput for a given network topology having static traffic on all the possible disjoint multiple paths. Then they proposed new optimized Interference Aware Pruned Two Path (IA-P2P) topology control scheme which used their network optimization model and pruned some links in the physical topology to select best two among multiple completely disjoint paths for each owner.

Some researches work on directly improving the network throughput, or balancing the throughput of different gateways The work in [6] constructed a forest for wireless networks, such that each tree carries similar traffic load. The work in [7] presented a topology control algorithm for WMNs to maximize bandwidth, with the assumption of directional antennas and zero interference. In [8], they proposed a Capacity-Optimized Cooperative (COCO) topology control scheme to improve the network capacity in MANETs by jointly considering both upper layer network capacity and physical layer cooperative communications.

Transmission power control [9] is widely known as an effective way to improve the throughput of wireless networks. Nodes transmit at appropriate power levels, such that they can guarantee the transmissions at appropriate date rate, without interfering too many other nodes. The network throughput will be enhanced, due to more concurrent transmissions. Rate adaptation is a mechanism unspecified by the 802.11 standards, yet critical to the system performance by exploiting the multi-rate capability at the physical layer. In [10], authors investigated the joint optimization of channel allocation, power control and routing under signal-to-interference-and-noise ratio (SINR) model for multi-channel multi-radio WMNs. They applied bio-inspired optimization techniques for channel allocation and power control. Rate adaption improves the network throughput in another direction. IEEE802.11 protocol family (IEEE802.11a/b/g) [11], [12] can provide multiple data rates, by using different PHY layer modulation schemes. Each modulation scheme can support a certain level of data rate, for the receivers within the transmission range. The transmission range is determined by the pair of the transmission power level and the modulation scheme. Auto rate fall back (ARF) [13] proposed to dynamically select a modulation scheme based on the channel condition, to optimize the network performance.

So far, there is no published work that considers how to meet the overall QoS requirements through topology control in wireless mesh networks. In this paper, we address the problem of topology control joint power adjustment that can meet the QoS requirements and the total throughput of system is maximized.

\section{PROBLEM Formulation}

The mesh network discussed in this paper consists of a set of mesh routers, some of which are gateway routers that have wire connection to the Internet. We simply call gateway routers gateways and non-gateway routers nodes. The set of nodes in the network is represented by a set $V$. The communication link is bi-directional and all traffic is to/from the Internet. We consider both uplink traffic and downlink traffic. The uplink traffic demand of node $\mathrm{v}$ is denoted as $\lambda_{V}^{\mathrm{up}}$, the downlink traffic demand of $\mathrm{v}$ is denoted as $\lambda_{V}^{\mathrm{dn}}$. The total traffic demand of a node $\mathrm{v}$, denoted by $\lambda_{V}$, is

$$
\lambda_{\mathrm{v}}=\lambda_{\mathrm{v}}^{u p}+{ }_{\mathrm{v}}^{d n}
$$

Let $\alpha_{\mathrm{v}}$ denote the traffic delivery ratio of node $\mathrm{v}$, $0 \leq \alpha_{\mathrm{v}} \leq 1$. That is, for node $\mathrm{v}$, only $\alpha_{\mathrm{v}} \lambda_{V}$ of end-user's traffic is guaranteed to be delivered to Internet due to the limited bandwidth of the system. The QoS requirement we concern is that we want to guarantee each node at least have minimal traffic delivery ratio $\alpha^{*}$, that is, for each node $\mathrm{v}$, we want to satisfy that at least $\alpha^{*} \lambda_{\mathrm{v}}$ of end-user's traffic must be guaranteed to be delivered to/from the Internet.

Our goal is to construct a logical topology that allows each node to route their end-users' traffic to the Internet via the gateways, and assign the transmission power for each node, such that each node's minimal traffic delivery ratio is fulfilled. Since we only consider a single path routing from a node to a gateway, the routes of all nodes to gateways form a forest and each tree in the forest is rooted from a gateway. The end-users' traffic is merged at the tree nodes who further pass the traffic to their parent nodes toward the gateway. Let $\mathrm{T}_{\mathrm{v}_{\mathrm{i}}}$ denote the subtree rooted at node $v_{i}$. And we use $1_{v_{i}}$ denote the nondirectional link connecting subtree $\mathrm{T}_{\mathrm{v}_{\mathrm{i}}}$ to its parent node, as shown in Fig. 1.

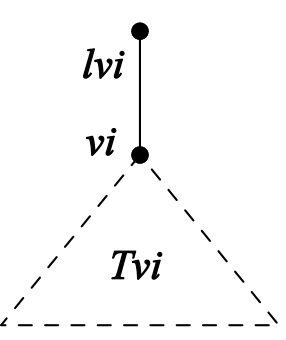

Fig. 1. Node $\mathrm{v}_{\mathrm{i}}$, its corresponding link $1_{\mathrm{v}_{\mathrm{i}}}$, and its subtree $\mathrm{T}_{\mathrm{v}_{\mathrm{i}}}$.

The uplink from $v_{\mathrm{i}}$ to its parent, is denoted as $I_{v_{\mathrm{i}}}^{u p}$, and the downlink from $\mathrm{v}_{\mathrm{i}}$ 's parent to $\mathrm{V}_{\mathrm{i}}$, is denoted as $I_{V_{i}}^{\mathrm{dn}}$. The corresponding uplink and downlink traffic are denoted as $\mathrm{L}^{\mathrm{up}}\left(\mathrm{v}_{\mathrm{i}}\right)$, and $\mathrm{L}^{\mathrm{dn}}\left(\mathrm{v}_{\mathrm{i}}\right)$ respectively. Thus

$$
\begin{aligned}
& L^{u p}\left(v_{i}\right)=\sum_{V_{j} \in T_{V_{i}}} \alpha_{V_{j}} \lambda_{V_{j}}^{u p} \\
& L^{\mathrm{d} n}\left(V_{i}\right)=\sum_{V_{j} \in T_{V_{i}}} \alpha_{V_{j}} \lambda_{V_{j}}^{d n}
\end{aligned}
$$


The link's data rate is determined by the transmission power of the sender, and the distance between two end-nodes. Let us denote $P_{v}$ as the transmission power of $v$, and $d(e)$ as the distance between two end-nodes of link $e$. The data rate of uplink $I_{V}^{u p}, \mathrm{~B}\left(I_{V}^{u p}\right)$, can be represented as a function

$$
B\left(I_{V}^{u p}\right)=f\left(P_{V}, d\left(I_{V}^{u p}\right)\right)
$$

Similarly, the data rate of a downlink $l_{V}^{\mathrm{dn}}$ is

$$
B\left(I_{V}^{\mathrm{dn}}\right)=f\left(P_{V}, d\left(I_{V}^{d n}\right)\right)
$$

where $v^{\prime}$ is v's parent node. Notice that, for the same link, its uplink data rate may not be same as its downlink date rate, as the transmission power of its two end-nodes may not be same.

The interference range of a node $\mathrm{v}$ is determined by its transmission power. A node $\mathrm{x}$ is interfered by a node $\mathrm{y}$, if the received signal at $\mathrm{x}$ from $\mathrm{y}$ exceeds a threshold $\beta$, that is,

$$
\mathrm{G}(y, x) P_{y} \geq \beta
$$

where $\mathrm{G}(\mathrm{y}, \mathrm{x})$ is the signal attenuation rate from $\mathrm{y}$ to $\mathrm{x}$.

Two nodes interfere with each other when their distance is within the interference range. Since we assume communication links are bi-directional, two links $1_{1}$ and $l_{2}$ interfere with each other iff one end-node of $l_{1}$ interferes with one end-node of $1_{2}$. For link $1_{V_{i}}$, we define its collision set as a set of links that interfere with it, including itself, and denote this collision set as $\mathrm{I}\left(1_{V_{i}}\right)$.

In wireless communication, two links that interfere with each other cannot be active at the same time due to the signal interference. We consider the most conservative case that no two links in the same collision set can be active at the same time in order to guarantee successful transmissions. That is, the total active time for links in the same collision set per unit time cannot exceed one. In each unit of time, the uplink traffic of $1_{V_{i}}$ is active for a time duration of

$$
\frac{\mathrm{L}^{u p}\left(V_{i}\right)}{B\left(I_{V_{\mathrm{i}}}^{u p}\right)}
$$

and the downlink traffic is active for

$$
\frac{\mathrm{L}^{\mathrm{dn}}\left(V_{i}\right)}{B\left(I_{V_{\mathrm{i}}}^{d n}\right)}
$$

Let $\mathrm{L}_{I\left(l_{V_{i}}\right)}$ be the collision load of link $1_{V_{i}}$. It is defined as

$$
\mathrm{L}_{I\left(I_{v_{i}}\right)}=\sum_{I_{v_{k}} \in I\left(I_{v_{k}}\right)} \frac{L^{u p}\left(v_{k}\right)}{B\left(I_{V_{\mathrm{k}}}^{u p}\right)}+\frac{L^{d n}\left(V_{k}\right)}{B\left(I_{V_{\mathrm{k}}}^{d n}\right)}
$$

Based on above analysis, we can easily get the following constraint,

$$
\mathrm{L}_{I\left(l_{v_{i}}\right)} \leq 1
$$

Thus, the traffic delivery ratio of node $\mathrm{v}_{\mathrm{i}}$ should fulfill requirement

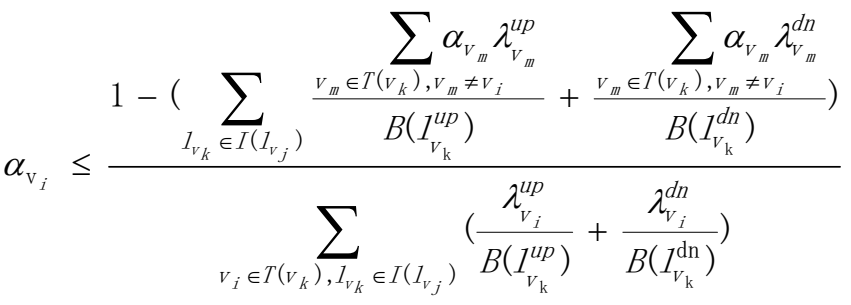

$$
\begin{aligned}
& , \forall I_{V_{j}} \in \bigcup_{V_{i} \in T\left(V_{p}\right)} I\left(I_{V_{p}}\right)
\end{aligned}
$$

The capacity of our concern is the maximal system total throughput. It's the sum of each node's throughput. That is,

$$
\mathrm{TP}=\sum_{V \in V} \alpha_{V}\left(\lambda_{V}^{u p}+\lambda_{V}^{d n}\right)
$$

We have to guarantee the QoS requirement, that is for each node $\mathrm{v}$, the delivery ratio must exceed the bound $\alpha^{*}$.

$$
\forall \mathrm{v} \in V, \alpha_{V} \geq \alpha^{*}
$$

Given a network of nodes in $\mathrm{V}$ and their locations, our task is to construct a set of routing trees out of $\mathrm{V}$, whose roots are the gateways, to route end-users' traffic from each node to the Internet via the gateways, such that each node's delivery ratio satisfies the (13) constraint, and the system total throughput is maximized.

\section{ALGORITHM DESIGN}

Our algorithm constructs the topology in a greedy way. Each time a gateway selects a candidate node with the closest relative distance. Each candidate selects a parent node, and a power level to connect to it. Among all candidates, the one which generates a topology of the minimal maximal collision load is added to the forest. The procedure repeats until all nodes are added to the forest.

We define the relative distance from a node to a gateway in a wireless mesh network with multiple gateways as follows. We construct a graph $\mathrm{G}(\mathrm{V}, \mathrm{E})$. There is one edge $(\mathrm{u}, \mathrm{v})$ in $\mathrm{E}$, when $\mathrm{u}$ and $\mathrm{v}$ can reach each other with the maximal transmission range. This transmission range is the one of nodes that transmits at the highest power level with the lowest data rate. Let $h_{u, v}$ be the number of hops between node $u$ and $v$, $\mathrm{u}$ and $\mathrm{v}$ in $\mathrm{V},(\mathrm{u}, \mathrm{v})$ in E. Suppose there are M gateways in the system, denoted by $\mathrm{W}=\left\{\mathrm{g}_{1}, \mathrm{~g}_{2}, \ldots, \mathrm{g}_{\mathrm{M}}\right\}$. For each node $\mathrm{u}$, we define a vector of hop-numbers between $\mathrm{u}$ and all the gateways, denoted as $\mathrm{H}_{\mathrm{u}}$ :

$$
H_{u}=<h_{u, g_{1}}, h_{u, g_{2}}, \ldots, h_{u, g_{M}}>
$$

For each gateway $\mathrm{g}_{i} \in W$, the vector of hop-counts to all gateways is denoted as $H_{g_{i}},\left(h_{g_{i}, g_{i}}=0\right)$ :

$$
H_{\mathrm{g}_{i}}=<h_{g_{i}, g_{1}}, h_{g_{i}, g_{2}}, \ldots, h_{g_{i}, g_{M}}>,\left(h_{g_{i}, g_{i}}=0\right)
$$

We define the relative distance between a node $\mathrm{u}$ and $\mathrm{a}$ gateway $\mathrm{g}_{\mathrm{i}}$ as the distance between vector $\mathrm{H}_{\mathrm{u}}$ and the $H_{\mathrm{g}_{i}}$, denoted by $\mathrm{d}\left(\mathrm{u}, \mathrm{g}_{\mathrm{i}}\right)$. The cosine distance [14] is known as an 
useful metric to measure the distance between two vectors. The cosine distance between two vectors $\vec{P}$ and $\vec{Q}$ is defined as the angle between them, which is

$$
\operatorname{angle}(\vec{P}, \vec{Q})=\cos ^{-1}\left(\frac{\vec{P} \cdot \vec{Q}}{|\overrightarrow{P \| Q}|}\right)
$$

The relative distance is thus

$$
\begin{aligned}
d\left(u, g_{i}\right) & =\operatorname{angle}\left(H_{u}, H_{g_{i}}\right) \\
& =\cos ^{-1}\left(\frac{\sum_{g_{i} \in W} h_{u, g_{j}} h_{g_{i}, g_{j}}}{\sqrt{\sum_{g_{j} \in W} h_{u, g_{j}}^{2}} \sqrt{\sum_{g_{j} \in W} h_{g_{i}, g_{j}}^{2}}}\right)
\end{aligned}
$$

$T_{\mathrm{g}_{\mathrm{i}}}=\left\{g_{i}\right\}, g_{i} \in W$

$\mathrm{N}_{\mathrm{g}_{\mathrm{i}}}=\left\{\right.$ neighbors of $\left.g_{i}\right\}, g_{i} \in W$

$N=\{$ all non-gateway nodes, nodes' delivery ratio are initialized 0$\}$

$\alpha^{*}$ as the minimal traffic delivery ratio bound

while $\mathrm{N} \neq \phi$ do

for all $g_{i} \in W$ do

find $u_{u_{i}} \in T_{g_{i}}$ with $\min d\left(u_{i}, g_{i}\right)$

for all $p_{i} \in T_{g_{i}},\left(u_{i}, p_{i}\right) \in E$ do

for all possible pair of power level $\mathrm{P}_{u_{i}}$ for $u_{i}$, and $\mathrm{P}_{\mathrm{p}_{i}}$ for $p_{i} \mathbf{d o}$

calculate the corresponding delivery ratio of node $u_{i}$,

$$
\text { if } \alpha_{u_{i}}<\alpha^{*} \text { then }
$$

adjust other nodes' delivery ratio in collision set until node $u_{i}$ 's delivery ratio satisfies the QoS constraint

$$
\text { end if }
$$

\section{end for}

\section{end for}

find $p_{i} \in T_{g_{i}}$ with max system total throughput if

connecting $u_{i}$ to $p_{i}$

\section{end for}

if all candidates' can't be adjusted then

no valid topology can satisfy the QoS requirement, exit else

connect $u_{i}^{*}$ to $p_{i}^{*}$ with the power level $P_{u_{i}}^{*}$ and $P_{p_{i}}^{*}$

$/$ *denote $\left(\mathrm{u}_{\mathrm{i}}^{*}, \mathrm{p}_{\mathrm{i}}^{*}\right)$ as the $\left(u_{i}, p_{i}\right)$ that achieves max

system total throughput if connecting $u_{i}^{*}$ to $p_{i}^{*} * /$

\section{end if}

$\mathrm{T}_{\mathrm{g}_{\mathrm{i}}^{*}}=T_{g_{i}^{*}}+\left\{\mathrm{u}_{\mathrm{i}}^{*}\right\}$

$\mathrm{N}_{\mathrm{T}_{\mathrm{g}_{\mathrm{i}}^{*}}}=\mathrm{N}_{\mathrm{T}_{\mathrm{g}}^{*}}-\left\{\mathrm{u}_{\mathrm{i}}^{*}\right\}+\left\{\right.$ non - tree neighbors of $\left.u_{i}^{*}\right\}$

$N=N-\left\{u_{i}^{*}\right\}$

\section{end while}

output $\left\{\mathrm{T}_{\mathrm{g}_{i}} \mid g_{i} \in W\right\}$ as the network topology

Fig. 2. Joint power and topology control algorithm
Based on the above definition, a smaller $\mathrm{d}\left(\mathrm{u}, \mathrm{g}_{\mathrm{i}}\right)$ indicates that the distance vector from $u$ to all gateways has a better match of the distance vector from $\mathrm{g}_{\mathrm{i}}$ to all other gateways, i.e., $\mathrm{u}$ is relatively closer to $\mathrm{g}_{\mathrm{i}}$ than to other gateways. It means $\mathrm{u}$ is more urgent, compared with other nodes whose relative distance to $\mathrm{g}_{\mathrm{i}}$ is longer than $\mathrm{u}$, to be connected to the subtree of $g_{i}$. By connecting $u$ to the subtree of $g_{i}$ first, it allows the nodes who are also located nearby $\mathrm{g}_{\mathrm{i}}$ (in terms of Euclidean distance) but relatively closer to other gateways to connect to the other gateways for load balancing purpose at the later stage of the topology construction. If connecting these nodes to $g_{i}$ first as the SPT method does, later it would either force node $u$ to take much more hops to connect to other gateways (for load balancing), or simply connect $u$ to $g_{i}$ but causing load unbalancing.

The topology construction method works as follows. Initially, the forest consists of all the gateway nodes as roots of subtrees. In each round, each gateway $g_{i}$ finds the node $u$ whose $d\left(u, g_{i}\right)$ is the smallest among all the nodes. We evaluate all possible connections from $\mathrm{u}$ to one parent node $\mathrm{p}_{\mathrm{i}}$ in $T_{g_{i}}$.

For each possible connection, we evaluate all combinations of transmission power settings of $u$ and its parent node. Then we calculate node u's traffic delivery ratio $\alpha_{u}$ for each combination. If node u's traffic delivery ration doesn't satisfy the QoS requirement, that is $\alpha_{\mathrm{u}}<\alpha^{*}$, we have to make some adjustment. We should adjust the corresponding nodes' delivery ratio which have attached into the forest, such that node $u$ satisfy the QoS constraint.

Our adjust strategy is as this. First we sort the links in the collision set by descending order of hops between end-node to the gateway. Then we assign the candidate node's delivery ratio as the $\alpha^{*}$, we gradually adjust each link's end-node's delivery ratio until the candidate node's minimal delivery ratio is satisfied or not (that is, we adjust all node's delivery ratio in the collision set to the minimal delivery ratio, but the collision load per unit time is still larger than 1). According to (11), suppose the initial value of $\alpha_{\mathrm{v}_{i}}$ is $\alpha_{\mathrm{v}_{i}}^{0}, \alpha_{\mathrm{v}_{i}}$ is updated as

$$
\begin{aligned}
& \alpha_{\mathrm{v}_{i}}=\frac{1-\mathrm{L}_{I\left(I_{v_{j}}\right)}}{\sum_{V_{i} \in T\left(v_{k}\right), I_{v_{k}} \in I\left(l_{v_{j}}\right)}\left(\frac{\lambda_{V_{i}}^{u p}}{B\left(l_{V_{\mathrm{k}}}^{u p}\right)}+\frac{\lambda_{V_{i}}^{d n}}{B\left(l_{V_{\mathrm{k}}}^{\mathrm{dn}}\right)}\right)}+\alpha_{V_{i}}^{0}, \\
& \forall l_{V_{j}} \in I\left(l_{V_{j}}\right)
\end{aligned}
$$

We first adjust the link far away from the gateway than others for the sake of maximizing the system throughput, as far away from the gateway's end-node's traffic need more relay nodes. After all gateways find their most suitable candidates to be connected into their subtrees, the candidate that results in the maximal system total throughput will be selected and connected to the respective subtree. The process is repeated until all nodes are eventually connected to the subtrees (of the forest). The details of our main method and the adjustment strategy are shown in Fig. 2 and Fig. 3. 


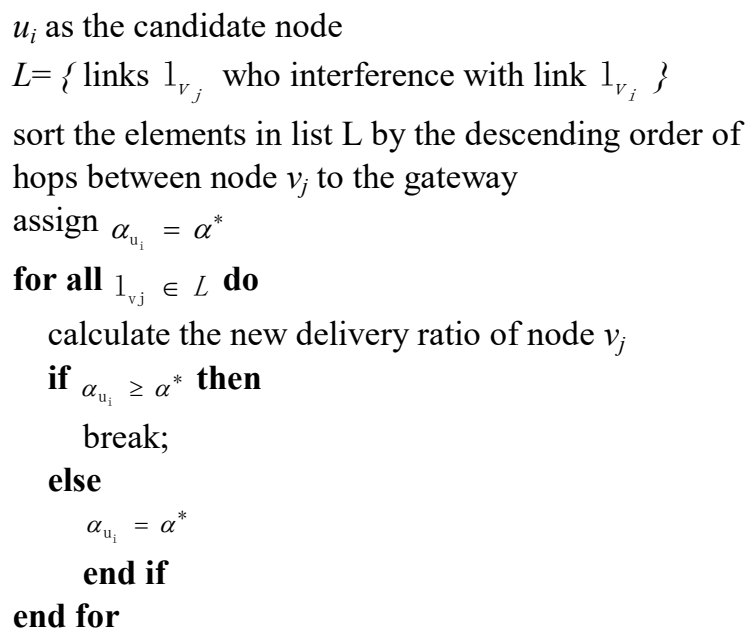

Fig. 3. Adjusting procedure in the collision set.

\section{PERFormance EVAlUation}

The simulations are conducted in a $300 \mathrm{~m} * 100 \mathrm{~m}$ region, with 100 non-gateway nodes. The channel capacity is set to $54 \mathrm{Mbps}$. Each routers has a total traffic demand of $1 \mathrm{Mbps}$, including $0.9 \mathrm{Mbps}$ downlink traffic demand and $0.1 \mathrm{Mbps}$ uplink traffic demand. The table of transmission range and interference range by transmission power and data rate is shown in Table I. The entry indicated by the pair of data rate and transmission power (TX power).

TABLE I: TRANSMISSION RANGE AND INTERFERENCE RANGE VS. TRANSMISSION POWER AND DATA RATE

\begin{tabular}{|c|l|l|l|l|l|l|l|}
\hline \multirow{3}{*}{ Range $(\mathrm{m})$} & Rate & \multicolumn{2}{|c|}{ TX power(dBm) } \\
\cline { 2 - 9 } & Mbps & 8 & 11 & 14 & 17 & 20 & 23 \\
\hline \multirow{5}{*}{$\begin{array}{c}\text { Transmission } \\
(\mathrm{m})\end{array}$} & 6 & 29 & 36 & 43 & 53 & 64 & 78 \\
\cline { 2 - 9 } & 9 & 20 & 24 & 29 & 36 & 43 & 53 \\
\cline { 2 - 9 } & 12 & 17 & 21 & 26 & 31 & 38 & 46 \\
\cline { 2 - 9 } & 18 & 15 & 18 & 22 & 27 & 33 & 41 \\
\cline { 2 - 9 } & 24 & 12 & 15 & 18 & 22 & 27 & 33 \\
\cline { 2 - 8 } & 36 & 10 & 12 & 14 & 17 & 21 & 26 \\
\cline { 2 - 8 } & 48 & 7 & 9 & 11 & 13 & 16 & 20 \\
\cline { 2 - 8 } & 54 & 7 & 8 & 10 & 12 & 15 & 18 \\
\hline Interference(m) & & 33 & 41 & 49 & 60 & 73 & 89 \\
\hline
\end{tabular}

We simulate three kinds of scenarios in this simulation, which are 1) scenarios of unevenly distributed nodes and evenly distributed gateways, 2) scenarios of evenly distributed nodes and unevenly distributed gateways, and 3) scenarios of uniformly distributed nodes and gateways. They are representative as different distributions can be classified into one of these three kinds of scenarios. We compare the performance of our method, named QOS_Power, with shortest path tree (SPT) method. We will investigate the impact of different system parameters, such as transmission range, different traffic delivery ratio bound and number of gateways, on the performance of algorithms.

\section{A. Scenario of Evenly Distributed Nodes and Unevenly Distributed Gateways}

The scenario of evenly distributed nodes and unevenly distributed gateways is shown in Fig. 4(a). The whole area is evenly divided into three subareas A, B, and C. Two gateways are placed at the top boundary, where gateway G1 is placed at the left corner and gateway G2 is placed at the center of subareas B. 60 nodes are uniformly distributed in subarea B, and all the other 40 nodes are uniformly placed in the subareas A and $\mathrm{C}$.

First we use our former paper's RCF method [3] in this scenario, and compute the response maximal traffic delivery ratio $\alpha$ as the max per node delivery ratio. Then we use different traffic delivery ratio bound to get the corresponding topology and system throughput. Through 50 times simulation, As shown in Fig. 4(b), system throughput generally decreases as the per node delivery ratio bound increases. When delivery ratio bound is 0.08 , System throughput decreases $36.7 \%$ than that 0 . As we should fulfill the QoS requirements of nodes far away from the gateways, and this decreases the delivery ratio of nodes near the gateways, so system throughput degrades. We consider a two hop scenario, node A directly connects to gateway $\mathrm{G}$, node $\mathrm{B}$ connects to $\mathrm{G}$ through node $\mathrm{A}$. Suppose the total capacity is 1 Mbps, both node A and B's traffic demand are 1 Mbps. When delivery ratio bound is 0.1 , then we can assign node $\mathrm{A}$ 's ratio as 0.8 and B's ratio as 0.1 to achieve the maximal system throughput, the total throughput is 0.9 Mbps. But When the delivery ratio bound increases, as to 0.2 , then we can assign node A's ratio as 0.6 and B's ratio as 0.2 , thus the total throughput is 0.8 . As nodes near the gateway should relay the traffic of nodes far away from the gateway, so if the delivery ratio bound increases, the system throughput decreases.

QOS_Power outperforms the SPT method in all delivery ratios bound. When delivery ratio bound is 0.06 , SPT method cannot satisfy this QoS requirement. But QOS_Power method can support up to 0.08 per node delivery ratio bound. On average, the QOS_Power achieves around 84.6\% higher throughput than the SPT method. This is because, in this scenario, the SPT connects almost all nodes in subarea A and $\mathrm{B}$ to $\mathrm{G} 2$, thus leads a unbalanced forest, and degrades the system throughput.

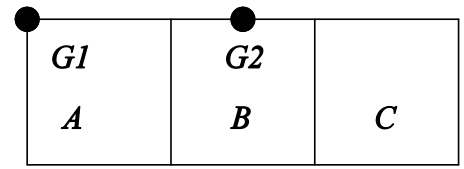

(a) Node distribution

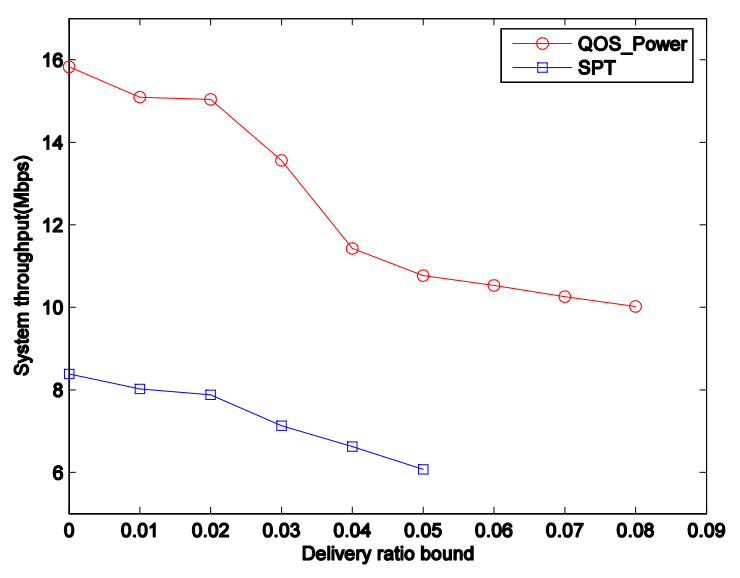

(b) Throughput

Fig. 4. Network of evenly distributed nodes and unevenly distributed gateways. 


\section{B. Scenario of Unevenly Distributed Nodes and Evenly Distributed Gateways}

The scenario of unevenly distributed nodes and evenly distributed gateways is shown in Fig. 5(a). The whole area is evenly divided into four subareas A, B, C and D. Three gateway $\mathrm{G} 1, \mathrm{G} 2$, and $\mathrm{G} 3$ are placed along the top boundary of the area, where G1 and G3 are at the two sides and G2 at the middle. The gateways are placed at the boundary to ensure there exists some long paths, that is, paths of hop-numbers more than 2, to ensure that there are some nodes outside the interference area of some gateways. The number of nodes in each subarea decreases from left to right, which is $40,30,20$, 10 nodes for subarea A, B, C and D respectively, and nodes are placed randomly in each subarea.

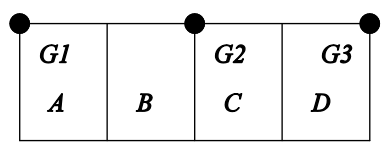

(a) Node distribution

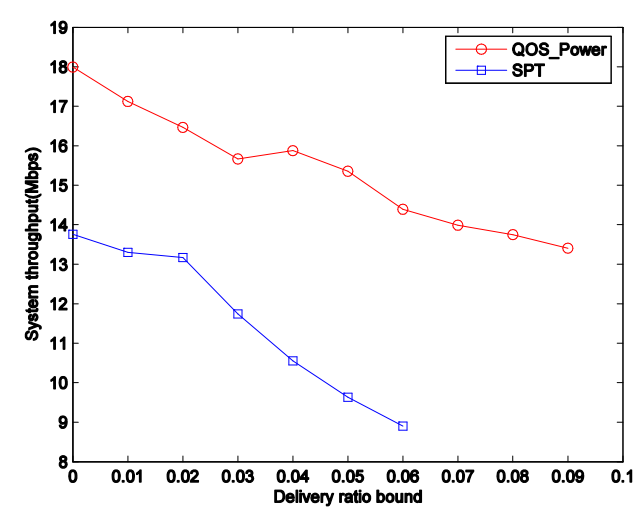

(b) Throughput

Fig. 5. Network of evenly distributed nodes and unevenly distributed gateways.

We can observe that QOS_Power outperform in each traffic delivery ratio bound. Both methods' system throughputs decrease as the increase of delivery ratio bound.

\section{Scenarios of Uniformly Distributed Nodes and Gateways}

In this subsection, we simulate a scenario of uniformly distributed nodes and gateways. We place 100 non-gateway nodes and $\mathrm{K}$ gateways uniformly in a $300 \mathrm{~m} * 100 \mathrm{~m}$, where $\mathrm{K}$ varies from 3 to 9 .

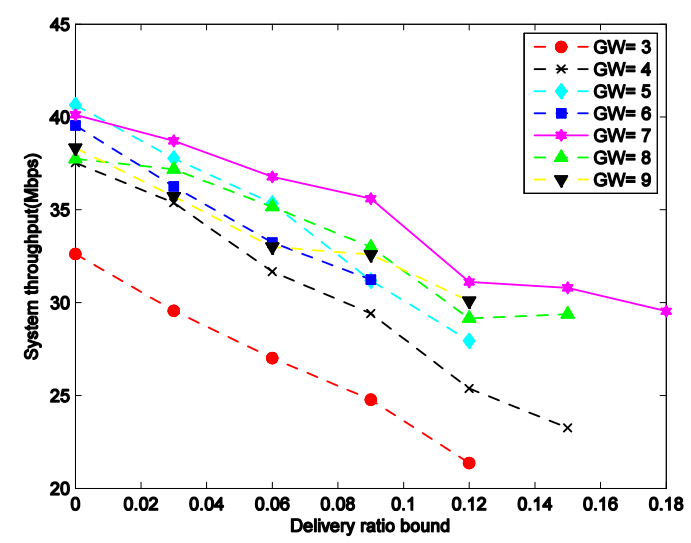

Fig. 6. System throughput vs. different delivery ratio bound in different gateways scenarios.
As shown in Fig. 6, system throughput decreases as the increase of per-node delivery ratio bound. System throughput increases as the increase of the number of gateways and get the pick when the number of gateways if 7 , because both the number of nodes assigned to trees rooted at each gateway and relay traffic decrease as the increase of the number of gateways. But when the number of gateways increases beyond 7 , system throughput decreases as add more gateways. When the number of gateways is small, the gateways are far away from each other and do not interfere with each other. In this case, the number of nodes per gateway decreases. When the number of gateways increases beyond a certain threshold, in such a case, one link may interference two or more adjacent gateways. This may lead the system performance degrades.

\section{CONCLUSION}

In this paper, we investigated a joint power and topology control method to construct a high throughput topology satisfying QoS requirement for wireless mesh networks. We formulate the network capacity, which is aware of rate adaptation and both uplink and downlink traffic. Then we propose a greedy method to construct a routing forest and assign nodes' transmission power, both consider the load balancing and throughput maximizing. We compare the algorithm with different QoS constraint in different scenarios. The simulation results have shown that our algorithm outperform the SPT method in different scenarios and achieve higher system throughput.

\section{REFERENCES}

[1] I. F. Akyildiz and X. Wang, "A survey on wireless mesh networks," IEEE Communications Magazine, vol. 43, no. 9, pp. 23-30, Sept. 2005.

[2] D. D. Murugeswari et al., "A multi-objective evolutionary algorithm based qos routing in wireless mesh networks," Applied Soft Computing, vol. 40, no. C, pp. 517-525, 2015.

[3] J. Zhang, B. Wang, and X. Jia, "Relative-closest connect-first method for topology control in wireless mesh networks," in Proc. IEEE Globecom '09, 2009.

[4] M. E. A. Shankara and B. Bhagavan, "Topology control in wireless mesh networks," in NUST-SEECS, 2014.

[5] T. H. Liu and W. J. Liao, "Location-dependent network performance and design strategies for wireless mesh networks," in IEEE INFOCOM, Phoenix, AZ, USA, April 2008, pp. 2243-2251.

[6] B. He, D. Sun, and D. P. Agrawal, "Diffusion based distributed internet gateway load balancing in a wireless mesh network," in Proc. IEEE Globecom '09, 2009.

[7] Y. Bejerano, S. Han, and A. Kumar, "Efficient load-balancing routing for wireless mesh networks," Computer Networks, vol. 51, no. 10, pp. 2450-2466, July 2007.

[8] K. P. P. Babu, "Improves network capacity using topology control in wireless network," International Journal of Advanced Research in Computer and Communication Engineering, vol. 4, pp. 184-186, 2015.

[9] K. P. Kim and D. Tipper, "Improving the performance of multi-hop wireless networks by selective transmission power control," Journal of Information \& Communication Convergence Engineering, vol. 13, no. 1, pp. 7-14, 2015.

[10] Y. J. Jia and J. Chen, "Joint topology control and routing for multi-radio multi-channel WMNS under SINR model using bio-inspired techniques," Applied Soft Computing, vol. 32, pp. 49-58, 2015.

[11] Wireless LAN Media Access Control (MAC) and Physical Layer (PHY) Specifications, IEEE Standard 802.11, 1999.

[12] Wireless LAN Media Access Control (MAC) and Physical Layer (PHY) Specifications-Amendment 1: High-speed Physical Layer in the 5 GHz band, 1999. 
[13] G. Holland, N. Vaidya, and P. Bahl, "A rate-adaptive mac protocol for multi-hop wireless networks," in ACM MOBICOM, 2001.

[14] G. Qian, S. Sural, Y. Gu, and S. Pramanik, "Similarity between euclidean and cosine angle distance for nearest neighbor queries," in Proc. the 2004 ACM symposium on Applied Computing, New York, NY, USA: ACM, 2004, pp. 1232-1237.

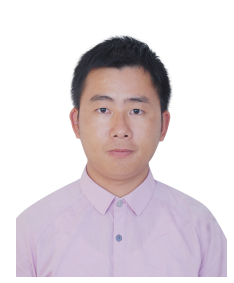

Kunxiao Zhou received his B.Sc. degree in computer science from Central China Normal University in 2004, and M.Eng and D.Eng degrees in computer science from Wuhan University, China, in 2006 and 2012, respectively. He received his D.Phil degree in Computer Science from City University of Hong Kong in 2013. He is currently an assistant professor in School of Computing at Dongguan University of Technology, China. His research interests include wireless ad hoc networks, wireless mesh networks, and wireless sensor networks.

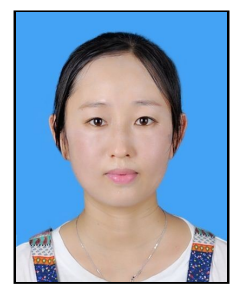

networks.

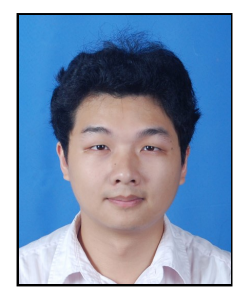

Hui Zhao received her B.Sc. degree in computer science from Inner Mongolia Agricultural University in 2007, and M.Eng and D.Eng degrees in computer science from University of Electronic Science and Technology of China, China, in 2009 and 2013, respectively. She is currently an assistant professor in School of Computing at Dongguan University of Technology, China. Her research interests include vehicular ad hoc networks, and wireless sensor

Xing Tang received his BEng degree from the School of Electronic Information in 2006 and DEng in degree from the School of Computer at Wuhan University, China in 2012, and his $\mathrm{PhD}$ degree in computer science from City University of Hong Kong in 2015. $\mathrm{He}$ is currently an assistant professor with the School of Computer Science and Technology, Wuhan University of Technology. His research interests include distributed systems and wireless networks. 